\author{
CORPUS \\ Corpus \\ 22 | 2021 \\ Du recueil à l'outillage des corpus oraux : comment \\ accéder à la variation?
}

\title{
Des discours authentiques singuliers aux scénarios de formation pour les médecins : quelle méthode d'exploitation pragmatique du corpus DECLICS2016?
}

From authentic original speech to pedagogical medical conversation scenarios: which kind of methodological pragmatic exploitation of DECLICS2016 corpus?

Emmanuèle Auriac-Slusarczyk et Aline Delsart

\section{OpenEdition}

Édition électronique

URL : https://journals.openedition.org/corpus/5960

DOI : $10.4000 /$ corpus.5960

ISSN : 1765-3126

Éditeur

Bases; corpus et langage - UMR 6039

\section{Référence électronique}

Emmanuèle Auriac-Slusarczyk et Aline Delsart, « Des discours authentiques singuliers aux scénarios de formation pour les médecins : quelle méthode d'exploitation pragmatique du corpus

DECLICS2016? », Corpus [En ligne], 22 | 2021, mis en ligne le 15 février 2021, consulté le 04 mars 2022. URL : http://journals.openedition.org/corpus/5960; DOI : https://doi.org/10.4000/corpus.5960

Ce document a été généré automatiquement le 4 mars 2022.

(C) Tous droits réservés 


\section{Des discours authentiques singuliers aux scénarios de formation pour les médecins : quelle méthode d'exploitation pragmatique $\mathrm{du}$ corpus DECLICS2016?}

From authentic original speech to pedagogical medical conversation scenarios: which kind of methodological pragmatic exploitation of DECLICS2016 corpus?

Emmanuèle Auriac-Slusarczyk et Aline Delsart

\section{Contexte introductif}

1 La linguistique de ou sur corpus (Léon, 2008) prend sens, pour partie, dans ses applications. C'est le cas des analyses politiques, par exemple, fort utiles pour comprendre les phénomènes pragmatiques de distorsions possibles des opinions via un usage langagier singulier (Trognon \& Larrue, 1997). Les études de linguistes ont d'ailleurs peu à peu migré vers l'utilisation, par les journalistes, d'outillages facilitant leur accès aux techniques de fouille automatisée, via des logiciels dédiés, gratuits, libres de droit (Marchand, 1998). Les travaux de Mayaffre sont à cet égard exemplaires d'une application utile et judicieuse au champ de l'analyse politique (Mayaffre, 2012; Mayaffre, Pincemin \& Poudat, 2019, pour exemples). À l'heure où nous écrivons cet article, c'est-à-dire en pleine effervescence sanitaire mondiale de la Covid-19, la portée du corpus DECLICS2016 peut même être interrogée quant à ses retombées sur la politique de santé publique. Ainsi, pourquoi faire un corpus, et quel usage envisager dès sa conception?

2 Initialement et structurellement, le projet DECLICS ${ }^{1}$ (Dispositif d'Etude CLInique des Corpus en Santé), dans lequel s'inscrit notre contribution, visait à faire dialoguer différents 
partenaires : des laboratoires de SHS et des laboratoires de médecine, associant des chercheurs francophones (Poitiers, Rennes, Nancy-Metz, Genève, Lyon). La valeur ajoutée de la collaboration concourait à présenter un nouveau corpus verbal dédié, DECLICS2016 ${ }^{2}$, pour réfléchir sur les relations soignants-patients à l'hôpital (Blasco et al., 2019). La linguistique appliquée (Auriac-Slusarczyk \& Blasco, 2019) contraint à sortir de son confort pour étudier des milieux divers, variés. Qu'en est-il du milieu médical ? À l'international (notamment Angleterre, USA, Allemagne, Québec) puis en France, l'évolution historique des méthodes en médecine jusqu'à la loi de modernisation du système de santé de 2016 (Berthod-Wurmser et al., 2017) a engagé un infléchissement progressif du modèle paternaliste (Préau \& Siméone, 2018). Ce dernier, reposant sur la confiance en l'autorité médicale jusqu'à l'usage même du mensonge (Faizang, 2006), recule au profit du modèle biopsysocial (Billon-Descarpentries, 2000); la direction de l'OMS installe dès 2006 la notion de people-centredness. À noter qu'en Angleterre, les groupes Balint (1957), d'orientation psychanalytique, prônaient déjà la méthode des focus groups entre médecins pairs généralistes, intégrant la verbalisation du vécu par les patients. La France s'est distinguée, pour sa part, en s'appuyant davantage sur les associations de patients, investiguant le contexte hospitalier plus que la médecine générale (Bousquet \& Ghadi, 2017). Ainsi, la pertinence des SHS en médecine est reconnue (Louis-Courvoisier, 2015) et l'étude de la conversation située en contexte médical est régulièrement retenue (Piot, 2018), notamment en contextes de suivi de maladies chroniques (Do \& Bissières, 2018; Balcou-Debussche \& Autier, 2018; BalcouDebussche, 2016a/b).

Que peut apporter la linguistique contemporaine à l'aune de ce tournant en santé ? La linguistique ne cesse, de son côté, de roder ses méthodes, tant théoriquement qu'empiriquement. Pour ce qui concerne notre orientation pragmatique, allant de la philosophie du langage à l'actualisation des travaux d'Austin, plusieurs équipes ont fait école: les écoles genevoise, lyonnaise, nancéienne ont chacune développé des méthodes d'analyse de discours. Par ailleurs, au plan empirique, le développement de la linguistique de et sur corpus a occasionné un nombre impressionnant de travaux dont la revue Corpus se fait justement l'écho (Mellet, 2002). La question se décale alors un peu. Est-ce qu'une étude particularisée de paroles authentiques, recueillies, transcrites et analysées par des linguistes a des chances d'intéresser le monde professionnel de la médecine, sachant que depuis les années 90 le genre de la consultation médicale est abordé (Lacoste et al., 1993) à partir de matériau ad hoc (Batt \& Trognon, 2012, pour exemple) ? De quelle manière épauler les médecins ? Comment introduire auprès des médecins une vision de la linguistique renouvelant leur intérêt quasi exclusif pour le vocabulaire, en relevant plutôt les conséquences de leurs emplois verbaux au mot près (Mayaffre, 2017)?

\section{Cadre référentiel}

4 Adaptée à la spécificité du corpus DECLICS2016, qui comprend un dispositif expérimental impliquant des thérapeutes pour le suivi de malades chroniques, notre question centrale est la suivante: la linguistique contemporaine peut-elle aider à une reconfiguration communicationnelle de la relation médecin-patient (cf. BerthodWurmser et al., 2017, ci-dessus)? 


\subsection{La notion de corpus en linguistique : généralités}

En annonçant que nous abordons ce champ sous l'angle assumé des généralités, nous reprenons une citation, un peu longue figurant sur le premier volume de la revue Corpus :

[1]a notion de corpus paraît, de prime abord, assez simple et bien ancrée dans certaines traditions des sciences humaines et sociales, philologique ou juridique par exemple. Il s'agit d'un recueil, formé d'un ensemble de données sélectionnées et rassemblées pour intéresser une même discipline. Néanmoins, dans le champ linguistique, la notion s'est complexifiée au cours des dernières décennies en fonction de la diversité des pratiques et des objectifs assignés à la constitution et à l'exploitation des corpus. Or le moment semble venu d'expliciter ces pratiques, de les questionner et de tenter de mettre au jour leur impact épistémologique sur l'évolution de la discipline, tant il est vrai - comme l'écrit Damon Mayaffre - que la qualité première d'une démarche scientifique est d'être une démarche qui s'explicite. (Mellet, $2002: 1$ ).

6 Nous nous situons, quasiment 20 années plus tard, sur cette identique volonté de porter explicitement les choix de constitution, les méthodes d'exploitation, ce, sans cacher les soucis que rencontrent les linguistes dans le traitement interdisciplinaire de données en termes de retombées professionnelles (Kébir et al., 2020). C'est dans cette perspective épistémologique de la linguistique que nous situons les enjeux de cette contribution.

\subsection{L'exercice de la médecine : quel lien avec la linguistique ?}

7 Sur le volet professionnel, Lussier et Richard (2008) ont proposé un modèle assez normatif en contexte québécois (cité par Richard et al., 2010, cf. figure en annexe) polarisant la relation médecin-patient depuis le rôle de prise en charge jusqu'à la celui de facilitateur. Dès 1951, Parsons proposait une nomenclature, qui fut progressivement révisée, amendée, transformée (Szasz \& Hollender, 1956 ; Freidson, 1984 ; Emanuel \& Emanuel, 1992 ; Moumjid \& Carrère, 2000, cf. tableau en annexe). Par ailleurs, le modèle structurel de la consultation de type "étapiste " subsiste; il a servi de référence aux travaux des années 80-90 (Richard et al., 2010). Des 3 aux 10 étapes décrites et préconisées aux médecins en formation, on note un décalage avec la description que Cosnier et al. (1993) avaient construite à partir de données verbales authentiques. Différemment de Richard et Lussier, nous considérons, pour notre part, que la relation est au cœur du système d'interlocution (Trognon, 1995; Auriac, 2007), le social construisant la relation, l'intercompréhension savante dépendant du système de places interlocutives (Kerbrat-Orecchioni, 1987). Gagne-t-on à faciliter la parole des patients chroniques (Piot, 2018) ? Le linguiste peut-il, à ce niveau, être utile à former à la relation de soin?

\subsection{Linguistique et médecine : quels ponts, quelle(s) méthode ?}

8 Si l'enjeu de soin est important, on ne minimise pas les effets de distance entre la culture des médecins et celle des chercheurs en sciences sociales (Louis-Courvoisier, 2015); on se garde de toute naïveté. La pédagogie médicale n'est pas facile à installer (Boelen, 2014). Le modèle délibératif (Ericsson, 2004; Côté, 2015) pourrait paraitre le plus idoine aux prescriptions actuelles en santé (cf. plus haut). Nous prendrons comme 
ancrage la référence à la clinique de l'activité de Clot (2007), préférant déléguer la question du métier directement aux médecins hospitaliers spécialistes, plutôt qu'imposer un style pensé comme préférable a priori en matière de santé. Nous délaissons de même les modèles de formation à la consultation (Lussier \& Richard, 2008 ; Fournier \& Kerzanet, 2007) au profit d'une centration sur les souhaits des intéressés, proposant aux médecins des scénarios-type communicationnels à discuter. Rétroagir sur ses propres pratiques de consultations (Côté, 2015) via l'insertion de modules de SHS en $1^{\text {re }}$ année de formation en santé (Gaillard \& Lechopier, 2015) est possible, à partir de données linguistiques. Un premier focus group avec trois/quatre médecins experts fut envisagé à partir des données de DECLICS2016: nous les soumettions à des données verbales aménagées, en tentant de séparer le spécialiste de ses habitudes de raisonnement clinique médical (Jouquand et al., 2013; Audétat et al., 2011); nous changions le lexique employé, les références authentiques au traitement. Notre intérêt était à terme de pouvoir aider les internes en médecine à appréhender l'espace de la consultation médicale. On souhaitait court-circuiter le raisonnement médical activé via l'expérience professionnelle des spécialistes qui nous semblait peu transmissible en formation initiale. Mais, quelle marge de progression professionnelle peut-on attendre des représentations des médecins (Nguyen-Khac, 2017) quant à divers scénarios communicationnels?

\section{Petit et grand corpus : intérêt et limite du corpus DECLICS2016}

9 La notion de corpus n'est pas stabilisée. Doit-on considérer l'utilité d'un corpus via a) sa taille (Danino, 2018), sa dimension applicative à un secteur professionnel - le soin (Cosnier, Grosjean \& Lacoste, 1993), - l'éducation (Henrion-Latché \& Auriac-Slusarczyk, 2020), - les sciences politiques (cf. Trognon, Mayaffre, op. cit.), ou via b) son intérêt pour renouveler l'étude d'objets historiquement analysés - la métaphore (Cameron \& Deignan, 2003), ou encore via c) son ambition de dévoilement de nouveaux paramètres - la multimodalité, la gestuelle (Ferré, 2016a/b), enfin via d) son genre comme langue de spécialité révélant usages et contextes inédits (Blasco \& Cappeau, 2018; Blasco et al., 2019) ? L'ensemble de ces questions vise à caractériser l'objectif de constitution d'un corpus, cas de DECLICS2016. La qualification d'un corpus n'intervient en fait qu'une fois qu'il est finalisé et diffusé ; DECLICS2016 ne peut pour l'instant faire l'objet d'une diffusion, eu égard au caractère personnel des données contenues ${ }^{3}$, le partage des données restant restreint à un archivage au sein des $\mathrm{MSH}$ contributives ${ }^{4} / 5$ jusqu'à plus ample anonymisation.

\subsection{Optiques/visées et caractéristiques de DECLICS2016}

DECLICS2016 a été prévu pour permettre l'exploitation des données en tant que corpusbased, pour confirmer/infirmer des hypothèses préalables, mais aussi comme corpusdriven engageant à des procédures plus inductives, pour explorer les données sans $a$ priori (Williams, 2005: 13; Danino, 2020:5). C'est un corpus de paroles authentiques, enregistrées pour exploiter, si ce n'est des hypothèses de recherches préalables, les intentions qui ont prédéterminé sa constitution. Son exploitation prévoyait des études en syntaxe, suivant la méthode de corpus-driven (Advocat \& Blasco, à paraître) et en 
pragmatique, croisant dans ce cadre la double méthode corpus-based (Delsart, 2020) et corpus-driven (Advocat \& Delsart, 2018).

DECLICS2016 comporte un total de 31 heures 20 minutes réparties sur 42 séquences déclinées sous trois formats: 1) des consultations médicales entre un médecin hospitalier spécialisé et un patient, 2) des entretiens cliniques entre un thérapeute d'orientation psychanalytique et un patient, 3) des présentations cliniques (à la manière des présentations de Charcot, voir Clavurier, 2014) entre un thérapeute et un patient, placés face à un auditoire composé d'une équipe médicale spécialisée et de chercheurs en SHS. Comparativement à d'autres corpus (Chevalier, 2007, 2008; Chanet, 2003), c'est un petit corpus. Il pourra à terme entrer comme un corpus exploitable pour révéler des faits de langues liés à l'authenticité des paroles recueillies (Chevalier, 2008) et permettre des exploitations croisées (cf. Chevalier, 20076; Chanet, 20037). Il est pour l'heure à catégoriser comme corpus spécifique lié à une langue de spécialité, particularisant le genre de l'échange médical (Cosnier et al., 1993), composé d'emplois reliés a priori aux conditions singulières de sa situation de production (Roiné et al., à paraître).

L'exploitation de DECLICS2016 se fait actuellement au fil de l'eau, l'ensemble des données n'étant pas totalement transcrit, la rigueur scientifique de sa transcription rendant sa diffusion progressive pour être normée aux besoins de chaque étude ${ }^{8 / 9}$. Actuellement 23 séquences enregistrées sont transcrites, représentant 16 heures 14 minutes pour un total de 207442 mots (voir Tab 1. ci-après et nos annexes). Cinq services hospitaliers et leurs cinq médecins volontaires en neurologie, nutrition, maladies infectieuses, médecine interne et pédiatrie, contribuent à varier les conditions de recueil pour une hétérogénéité voulue. Cinq thérapeutes français d'orientation analytique ont pris en charge les entretiens et/ou présentations cliniques sur la base du volontariat.

\subsection{DECLICS2016 : caractéristiques d'un corpus volontairement varié et hétérogène}

13 La constitution du corpus fut initiée grâce au partenariat scientifique initial de deux services (neurologie/pédiatrie), mais les aléas des consultations n'ont pas permis de développer DECLICS2016 de manière linéaire. Les tableaux suivants (Tab. 1 et Tab. 2) renseignent sur la répartition des enregistrements (genre, participants, nombre de séquences).

Tableau 1. Composition du corpus DECLICS2016 au 3 avril 2020

\begin{tabular}{|l|l|l|l|l|l|l|}
\hline & Neurologie & $\begin{array}{l}\text { Maladies } \\
\text { infectieuses }\end{array}$ & Nutrition & $\begin{array}{l}\text { Médecine } \\
\text { interne }\end{array}$ & Pédiatrie & Total \\
\hline $\begin{array}{l}\text { Séquences } \\
\text { enregistrées }\end{array}$ & $\mathbf{1 8}$ & $\mathbf{8}$ & 9 & 5 & 2 & 42 \\
\hline Consultations & 15 & 3 & 6 & 1 & 1 & 26 \\
\hline Entretiens cliniques & 3 & & & & 0 & 3 \\
\hline
\end{tabular}




\begin{tabular}{|l|l|l|l|l|l|l|}
\hline $\begin{array}{l}\text { Présentations } \\
\text { cliniques }\end{array}$ & 5 & 3 & 4 & 1 & 13 \\
\hline
\end{tabular}

Le tableau en annexe détaille ces éléments en indiquant la durée de chaque séquence selon les services et les conditions d'enregistrement : consultations versus entretiens ou présentations cliniques.

Tableau 2. Participants du corpus DECLICS2016 ${ }^{10}$

\begin{tabular}{|l|l|l|l|}
\hline & Nombre de participants & Femmes & Hommes \\
\hline Thérapeutes & 5 & 2 & 3 \\
\hline Médecins & 10 & 6 & 4 \\
\hline Patients & 35 & 18 & 17 \\
\hline Aidants & 13 & 9 & 4 \\
\hline
\end{tabular}

15 Tel qu'initié au départ, DECLICS2016 met en regard une consultation et un entretien ou une présentation clinique conduits avec un même patient, ce que nous avons désigné par la notion de binômes (Delsart \& Marquès, 2019; Auriac-Slusarzyk \& Delsart, soumis), méthodologie qui permet de comparer les faits de langue entre médecins et thérapeutes. Six binômes sont accessibles, représentant un minimum pour des traitements statistiques. À défaut de davantage de verbalisations sous format de binômes, nos études linguistiques envisagent l'application de nos résultats au parcours de soin; nous vérifions dans quelle mesure la linguistique aide (ou non) le médecin spécialiste d'un domaine (neurologie, nutrition, pédiatrie, etc.) à transformer son regard sur son activité de consultation.

16 Nous forgeons ainsi l'hypothèse que la linguistique, en tant que SHS sert la médecine (voir notamment Auriac-Slusarczyk \& Blasco, 2019). Est-ce le cas?

\section{Un découpage du corpus DECLICS2016 avec extraction de marques a priori utiles}

17 Dans l'optique pragmatique de faire bénéficier aux médecins des avancées de la logique interlocutoire (voir Trognon, 2012, pour un historique circonstancié), sans prétendre embrasser néanmoins ce champ dans sa complexité, nous avons fait des choix discutables. Sont-ils les bons? Saisis entre la méthode corpus-based et corpus-driven, nous parions que certaines marques sont plus à même de comparer le discours des médecins vs thérapeutes. Il s'agit des marqueurs de régulation/structuration de la conversation (Auchlin, 1981) ou ponctuants verbaux (Vincent, 1993), des marqueurs indiquant les rapports de places (taxèmes, Kerbrat-Orecchioni, 1988), et ceux dénotant la cohérence discursive, en se focalisant exclusivement pour l'instant sur mais et donc (Delsat \& Marquès, 2019; Auriac-Slusarczyk \& Delsart, à paraître, soumis). Dans la lignée des travaux en logique interlocutoire, nous proposons l'analyse d'évènements 
interlocutoires (Delsart \& Auriac-Slusarczyk, 2020) que nous transformons en scénarios pédagogiques, en pointant des stratégies discursives locales déterminantes pour les soignants. Pour les médecins, nous tentons de typifier le genre de la consultation médicale à partir de ces scénarios. Nous avons ainsi exploré l'usage du lexique émotionnel (Auriac-Slusarczyk \& Delsart, 2020, soumis) et étudié les effets des hétérorépétitions locales (Delsart, 2020). L'exploration de DECLICS2016 est progressive.

\subsection{Les indicateurs sélectionnés et priorisés à destination du corps médical}

Nous discuterons des choix en conclusion: ici, nous présentons et illustrons la résultante de nos choix. Trois vignettes cliniques (en annexes) donnent l'aperçu exhaustif d'extraits initialement sélectionnés à destination des médecins, pour engager avec eux une discussion formative pour qualifier la communication en consultation médicale (cf. Kébir et al., 2020, op. cit.). Nous intégrons dans le corps de l'article un seul exemple (extrait 1., ci-dessous) montrant le relevé de marqueurs typiques largement connus des linguistes, les taxèmes (cf. Kerbrat-Orecchioni, 1987 ; Delsart \& Marquès, 2019).

Extrait 1. Rapport de places ${ }^{11}$

MED[0h01m07]: donc on va dire qu'on est à deux mois

PAT[0h01m09]: voilà tout à fait oui exactement

MED[0h04m09]: il y a pas d'horaire particulier

PAT[0h04m11]: non il $\mathbf{y}$ a pas d'horaire particulier voyez là là je ce matin j'ai eu des

palpitations quand j'ai fait la gym et puis là bon

AID[0h04m18]: à la gym tu t'approchais de la prise du traitement

PAT[0h04m22]: oui

MED[0h06m23]: ça ça peut être intéressant que vous voyiez une eritaboliste*

PAT[0h06m24]: oui voilà tout à fait

PAT[0h09m19]: moi je veux bien ça me gêne pas ça c'est vous qui décidez hein mais $\operatorname{MED}[0$ h09m22]: voilà on va essayer comme ça le Ratripex* il bouge pas

Légende : * voir plus bas (§ 4.1. et 4.2.) sur l'insertion de ces non mots.

Est-ce qu'un médecin mis face à cet extrait de corpus peut travailler la notion de rapport de places tel que les linguistes en font un outil d'analyse théorique et empirique?

Les extraits mis en annexes tentent pareillement de faciliter aux médecins l'accès à des interprétations basées sur des outils linguistiques. L'étude pragmatique des consultations a dégagé deux niveaux d'évènements interlocutoires touchant à la mise en scène (cf. Charaudeau, 1995): a) l'intercompréhension et b) les stratégies présupposées du médecin (cf. annexes). Ces deux catégories reposent sur le travail sur corpus du linguiste qui ne saurait être détaillé ici (Auriac-Slusarczyk, 2019 : 13-14).

21 Le médecin face à cet extrait-type est-il à même d'extraire des critères d'intelligibilité pour faire son métier (cf. Clot, 2007, op. cit.) ? La délimitation de scénarios formatifs prototypiques, basés sur ces évènements interlocutoires pré-analysés, paramètre des échanges caricaturaux censés aider le corps médical. En fait, nous avons dû réduire le champ d'interrogation des médecins à trois cibles, les confrontant 1) au rapport de places ; 2) à l'intercompréhension et 3) aux stratégies du médecin, laissant de côté les 
éléments portant sur les régulateurs ou ponctuants verbaux (oui, hum, bon, etc.) dont on sait pourtant qu'ils sont des indicateurs professionnels forts (ex : alors, voir Bouacha, 1981).

\subsection{Trois scénarios prototypiques visant des vignettes cliniques formatives}

Avons-nous conduit la bonne réduction? Le linguiste est-il bien placé pour s'adresser aux médecins? Comment, basé sur ses résultats, peut-il en faire profiter le médecin? Les questions suivantes sont adressées aux médecins: quel style d'échange préférezvous? Pourquoi ? Comment dénommeriez-vous le style de chaque scénario, l'attitude du médecin et du patient? Ces scénarios font-ils écho à votre pratique? Diriez-vous que vous adoptez un style constant ou variable, selon les patients, dans votre pratique? Si vous aviez à intervenir en formation auprès d'internes, préfèreriez-vous présenter l'un de ces scénarios, plutôt qu'un autre? Pourquoi? Il s'agissait de voir si l'échelle a) du marqueur clef, b) de l'évènement interlocutoire et c) du genre conversationnel était accessible au médecin. Et, conséquemment, savoir si les médecins repèrent la même chose, selon leur spécialité, leur âge, leur expérience professionnelle.

\section{La mise à l'épreuve auprès de médecins : focus group sur la base de notre matériau linguistique}

Le cadrage d'un premier focus group a impliqué trois médecins de spécialité, âge et expérience professionnelle variés portant les objectifs suivants : a) recueillir leur avis professionnel sur les pratiques du métier; b) discuter la qualité de communication entre médecin et patient et c) dialoguer sur une formation utile aux jeunes médecins. Nous indiquons ce que nous avons considéré comme écueils vs réussites quant à former à partir des paroles authentiques issues de DECLICS2016 et analysées par le linguiste.

\subsection{Nos vignettes minimales caricaturales : constat d'une trop grande réduction}

Trois micro vignettes présentaient des extraits d'échanges sous forme d'acteurs-types caricaturaux : assentiment, opposant ou négociateur (extraits 2, 3 et 4). L'intérêt était de disposer de vignettes simplifiées croisant les indicateurs linguistiques et nos interprétations interlocutoires pour ouvrir les trois pistes prédéfinies: a) rapport de place, b) intercompréhension et c) stratégies du médecin (cf. § 3.1.).

Extrait 2, micro vignette, patient-type assentiment

PAT[0h09m19]: moi je veux bien + ça me gêne pas ça c'est vous qui décidez hein mais ; MED[0h09m22]: voilà on va essayer comme ça + le Azilect** il bouge pas

Extrait 3, micro vignette, patient-type opposant

PAT[0h16m55]: c'est pour ça que j'ai employé le terme d'idiopathie orpheline**; MED[0h16m58]: oui oui oui j'ai bien saisi + hein mais c'est idiopathie** ça veut pas dire ça

Extrait 4, micro-vignette, médecin-type négociateur

MED[0h10m00]: nous on peut refaire un point par exemple dans trois mois avec la prise de sang et de voir où ça en est + après pour le suivi comme vous disiez 
essayer que vous puissiez vous trouver vos propres solutions euh avec l'aide de mes collègues

Légende : les mots de jargon sont repérés par un double astérisque (voir le §4.2. suivant).

La réduction à ces trois micro vignettes nous a conduits à une mise en doute de ce support, confirmée ensuite par l'entretien mené avec des médecins (voir §5).

\subsection{L'impasse lexicale : comment faire?}

La présentation des extraits aux médecins a posé problème : devions-nous souligner les indicateurs linguistiques ou les faire découvrir aux médecins? L'emploi du jargon par les médecins repéré de longue date comme entravant potentiellement la compréhension (Turpin, 2002: 58) et maintenant le patient à distance de la sphère savante nous a conduits à remplacer les termes jargonnant (extrait 2, plus haut) par des non mots inventés (cf. extrait 1 , $\$$ 3.1., et annexes). Il s'avère très délicat d'interroger un spécialiste - neurologue, nutritionniste, infectiologue - ou un généraliste, en leur ôtant cet habitus de jargon; le linguiste ne maîtrise pas plus que le quidam l'usage des mots de spécialité médicale et notre inventivité a trouvé sa limite. Nous avons opté pour réintroduire le jargon médical initial, l'évocation de médications inventées dérangeant le médecin. Si nous avons préféré l'exploitation de paroles authentiques, transformer l'authenticité de propos au profit d'une formation reste possible mais discutable; les limites d'une exploitation de données, alternativement originales $v s$ adaptées, doivent questionner la formation.

\subsection{Interpréter des graphiques : consensus sur l'habitus expert des médecins}

27 Les médecins, de formation scientifique, sont rodés à la lecture interprétative de graphiques et sensibles aux données chiffrées. Cependant, une présentation chiffrée (Fig. 1) fonctionne-t-elle mieux que des extraits de parole? N'est-elle pas réductrice ? Engage-t-elle à la réflexion? La simplification chiffrée des données vaut-elle davantage car créditant la linguistique de méthodes d'apparence scientifique ou usuelles? 
Figure 1. Extrait présenté aux médecins rapportant le nombre d'occurrences brutes d'emplois de mais et donc comparant patient (PAT) et médecin (MED) sur deux cas cliniques authentiques -A/B-.

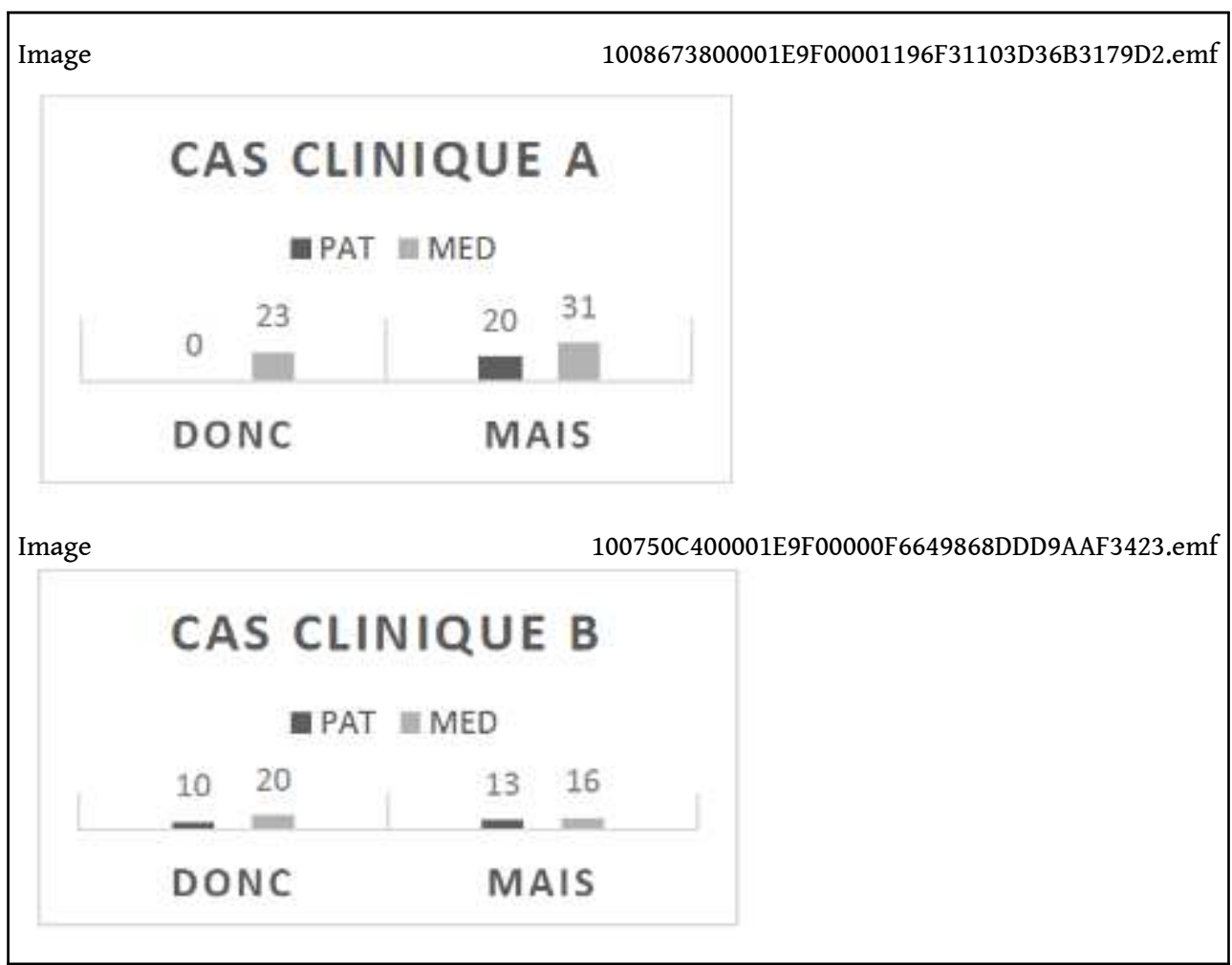

La prise de note effectuée in situ lors de la tenue du focus group révèle quelques caractéristiques saillantes des propos réactifs des médecins sur ces graphiques. Pour A, un médecin énonce son absence d'étonnement quant à l'emploi nul de donc, pointant comme évidente l'incapacité du patient à savoir interpréter ce qui se dit. Un autre médecin déclare la même absence d'étonnement, en notant qu'un patient préfère de toutes façons se distancier et laisser le médecin "s'occuper de tout». L'image du patient-type fonctionne extrapolée directement par les médecins et rendue par des propos tranchés : cela ne m'étonne pas du tout; je ne suis pas étonné12. L'approche par scénarios est pourrait-on dire alors nulle en effets formatifs : l'habitus diagnostique fait écran, l'évocation de l'expérience opacifie, il n'y a aucune mise en doute. Pour B, une image extrapolée de patient-type s'effectue pointant les équilibres d'emplois entre mais et donc. L'équilibre différent mais/donc engage le dialogue entre médecins : la conduite cohérente du discours par le truchement de mais est d'emblée attribuée au rôle de médecin, quand bien même est noté que l'emploi de mais ne destine pas à être contre le patient. Pour l'emploi de donc le patient respecterait son rôle de vérificateur de ce que le médecin a compris/transmis. Il semble n'y avoir aucune remise en cause des rôles connus d'avance de médecins et patients. L'expérience médicale voile toute nouvelle contextualisation possible des habitus diagnostiques (cf. Audetat et al., 2011): les médecins projettent leur expérience singulière dans l'usage de mais/donc et les apports du linguiste n'enclenchent aucun des processus formatifs réflexifs attendus. 


\subsection{Tenue du focus group avec des médecins : le court-circuit des faits de langues par les médecins} 2020, op. cit.) ne peut être exposé ici. Parmi les phases de ce déroulé, les consignes liées aux objectifs orientant sur les - a) rapport de place, b) intercompréhension, c) stratégies (cf. $\S 4.1$.), assorties à quatre extraits de paroles authentiques, furent soumis aux médecins (cf. annexes, vignettes 1, 2 et 3 ).

30 Nous avons proposé à trois médecins réunis de faire des commentaires spontanés et libres, discutant et revenant sur ce qui se disait entre eux. On leur présentait successivement quatre extraits d'échanges cliniques $^{13}$, qui, d'abord commentés librement, s'assortissaient ensuite de questions : 1. Que pensez-vous de cet extrait? 2. Que remarquez-vous du point des mots, des tournures de phrases? 3. À quoi êtes-vous sensibles? 4. Que retenez-vous de cet échange? Chaque extrait pensait l'exploitation de faits de langue orientée par notre étude linguistique préalable. Quelques points épineux ressortent des entretiens : les médecins notent et commentent a) l'absence de clarté, b) la protection/ défense du médecin, c) la taille des prises de paroles, d) la rupture du dialogue que les médecins déclarent comme étant à éviter. Les médecins renchérissent comme se contrarient : ce qui les frappe c'est l'impression d'un dialogue peu clair, sans axe défini dans ces paroles authentiques. Déroutés, ils cherchent des contextes d'interprétation, des appuis expérientiels et se disent surpris par le peu de paroles du médecin. Ils disent plausible la protection de tel médecin contre un envenimement de l'échange; dérangés par quelques propos assimilés à des menaces, ils déclarent ne jamais vivre ceci. Interrogés sur leurs propres modalités de prise de paroles, ils verbalisent a) leur désintérêt pour les histoires de vie des patients, b) la nécessité d'imposer un cadre/une orientation au patient, c) leur lutte contre les effets de ruptures du dialogue, enfin que d) leurs paroles ne sont pas réductibles aux extraits authentiques soumis.

De manière emblématique, ces trois médecins qui, du coup, ne se considèrent pas comme échantillon représentatif, court-circuitent la dimension linguistique proposée à leur attention; l'un dira : «je suis pas suffisamment acclimaté à repérer des mots clés, je repère mieux les non-dits ${ }^{14}$ ", et un autre indiquera que "le non verbal est une grande partie de la communication ». Le verbe est ainsi fui. L'un et l'autre affichent la même démotivation à décrypter les faits de langue, se dégageant de ce que les linguistes et ergonomes proposent; ils revendiquent être persuadés que la meilleure formation est celle du compagnonnage. Circonspects sur l'aspect réflexif que devraient suggérer les échanges authentiques qu'ils jugent distants de leurs habitus, ils disent que ces scénarios pourraient convenir à la formation des jeunes. Ils suggèrent alors une formation spécifiquement dédiée à la communication, en l'indiquant inutile pour eux ; ils disent la difficulté de se mettre dans des situations non vécues. Interrogés sur les simulations pratiquées en formation en internat de médecine, les médecins rapportent pourtant que ces simulations sont très stimulantes : ils expriment leur intérêt pour les jeux de rôles basés sur des cas cliniques, reconnaissent la similarité du procédé avec les cas soumis. Toutefois l'usage de vidéos, de films, d'emplois d'acteurs sous-entendus plus dynamiques ou de grilles d'observation pré-catégorisées leur paraît ne pas remplacer l'expérience clinique d'une carrière. Ils insistent alors soudainement sur l'importance des mots à la condition qu'on s'intéresse aux cas de diagnostics graves, cancers par exemple (cf. Saint-Dizier de Almeida, 2013). 
32 Au final, les médecins révèlent leur sensibilité au non verbal plus qu'au verbe et raillent les politiques de santé qui soumettent les jeunes internes à dire « la vérité » à tout prix. Un consensus semble acquis : soigner est une affaire de mots, et le verbe rejoint à ce niveau le choix politique. Mais les médecins ne sont pas prêts, en l'état des conditions proposées sur la base d'une exploitation du corpus DECLICS2016, à s'engager facilement à décrypter les/leurs/le discours.

\section{5. Éthique et exploitation de corpus : jusqu'où peut-on aller? Usage et partage du corpus DECLICS2016}

Les données de base que constituent les paroles authentiques enregistrées avec l'assentiment des patients, aidants, médecins et thérapeutes ne posent pas de souci à l'étude du linguiste, les chercheurs authentifiant le respect strict des conditions éthiques d'exploitation ${ }^{15}$. En revanche, les propos originaux et singuliers issus de la confrontation croisée entre médecins n'ont pu et ne peuvent être publiés ; les médecins ont confié verbalement leurs avis authentiques, au prix d'être assurés qu'on ne divulguerait cette vérité du verbe à quiconque. Ils n'ont donc pas consenti à la divulgation en l'état de leurs verbalisations. Nous avons dû gloser leurs propos, mais ne pouvons/pourrons en faire une étude trop fouillée. La linguistique de corpus est alors directement interpellée dans ses modalités de constitution et d'exploitation éthiques des paroles authentiques singulières. La forme de digression dans l'usage des données fournies par les linguistes, puis commentées par les médecins, semble ne pas fonctionner. La métaphore de la poupée russe où l'on pensait pouvoir emboiter des propos sur des propos pour gagner en application n'a pas totalement abouti. Cela interroge sur l'intérêt du projet DECLICS, ou le corpus DECLICS2016 se veut dédié à une linguistique appliquée (cf. Auriac-Slusarczyk \& Blasco, 2019). «À qui servent les corpus » reste une question fondamentale.

\section{Discussion générale}

Quand Kerbrat-Orecchioni étudie les faits de langue dans les boulangeries ou les boucheries (Kerbrat-Orecchioni, 2004), ce n'est pas pour former les petits commerçants français à être plus ou moins polis (Kerbrat-Orecchioni, 2001). Quand un corpus se destine à des retombées sociétales en termes de santé publique, sa constitution prend des tournures différentes, aux allures potentiellement ou pratiquement politiques. Notre contribution, en ce sens, questionne les linguistes, dont nous sommes, sur la qualité des études réalisées qui concernent de concert les conditions de constitution comme de portée d'un corpus. Avons-nous conduit les bons choix, la bonne réduction des faits de langue étudiés en scénarios, et su pré-adapter les temps d'échanges supposés formatifs auprès des médecins ? S'il est trop tôt pour conclure de manière décisive sur cet aspect, nous voudrions mettre en discussion certains éléments. Car comme le remarque C. Kerbrat-Orecchioni :

[...] de l'observation du fonctionnement de la politesse dans une situation aussi banale que l'achat d'une baguette de pain, il ressort: (1) Que loin d'être un phénomène marginal confiné dans quelques «formules" bien circonscrites, la politesse est en réalité diffuse et profuse, dans ce type de discours comme dans bien d'autres: près de la moitié du matériel produit dans les deux interactions qui 
viennent d'être analysées a une fonction moins transactionnelle que rituelle.

(Kerbrat-Orecchioni, 2001: 7). avons réalisées (cf. § 3.), constitue le terreau préalable d'une conclusion identique : du point de vue de la linguistique, la distribution, la diffraction des marques de discours, des tournures, des enchaînements interlocutoires rendent compte de la relation prototypique médecin-patient. Car, en matière de santé, il est question, aussi, de négocier (cf. Kerbrat-Orecchioni, 2004). En santé cependant, la levée de différends n'est pas sans conséquences pour la formation des médecins. Les stratégies, les changements de rôles, la construction des places discursives témoignent pour le linguiste d'une relation sociale et statutaire qui construit la relation patient-médecin. Toute politique de santé publique pensant les conséquences de cette relation de soin doit apercevoir qu'elle est verbalement tissée. Mais voilà, le médecin confronté aux faits linguistiques n'en tire pas nécessairement parti. Même si on sait la confrontation à des cas cliniques fructueuse en formation initiale en médecine (cf. Sommer \& Rider, 2014; Audétat, Laurin \& Sanche, 2011 ; cf. § 4.4), le passage d'une linguistique fondamentale à son usage académique en formation (cf. Millette, Lussier \& Goudreau, 2004) soulève des questions épineuses. Comment bloquer les habitus de raisonnements des médecins, et provoquer chez eux des interrogations sur leur expertise communicationnelle? Comment saisir les freins, leviers, obstacles, intérêts et motivations au changement ? La dé-contextualisation lexicale était-elle, même si nous l'avons abandonnée (cf. § 4.2.), une bonne idée? Faudrait-il informer les médecins des résultats en SHS avant la confrontation aux cas cliniques auxquels nous les avons soumis? A-t-on intérêt à présenter des cas similaires en réunion de service, aux internes seulement, à un collectif de médecins aux spécialités variées ? Peut-on faire envisager aux médecins une appropriation puis une forme d'utilisation de ces scénarios avec leurs internes? Est-ce souhaitable? Ne risque-t-on pas, en ce cas, des dérives de surutilisation, raccourci ou distorsion quant à l'interprétation de certains faits de langue mal appropriés (cas des équilibres mais/donc, cf. § 4.3.) ? A-t-on intérêt à utiliser les scénarios comparant des situations authentiques jugées somme toute artificielles (cf. §. 4.4. et 4.3.) ou provoquer des occasions de pénétrer en tant que linguiste les équipes médicales en terrain hospitalier? Toutes ces questions intéressent directement le linguiste, qui reste responsable de la portée de ses travaux. Nous espérons que cette contribution peut, modestement, engager la discussion à ce niveau.

\section{BIBLIOGRAPHIE}

Advocat O. \& Delsart A. (2018). « Regards croisés sur les données du corpus DECLICS2016 ", Colloque CA2LI, Lier Recherche et Formation professionnelle : les corpus à l'interface, 16 novembre 2018, UCA-MSH, Clermont Ferrand. 
Advocat O. \& Blasco M. (à paraitre). «Étude d'entretiens médicaux : procédés de densification et de réduction des formes linguistiques à l'oral ", in H. Gruet-Skrabalova et F. Spitzl-Dupic (éd.). Fonctions discursives des formes linguistiques réduites. Allemagne : Nodus, Münster.

Advocat O., Blasco M. \& Durif F. (2019). « Les entretiens entre patients et professionnels de santé : éléments de construction collective des discours ", ESASO 5(2) : 23-39.

Aubry R. \& Mallet D. (2008). « Réflexions et propositions pour la formation médicale ", Pédagogie médicale 9(2) : 94-102.

Auchlin A. (1981). « Mais heu, pis bon, ben alors voilà, quoi ! Marqueurs de structuration de la conversation et complétude », Cahiers de linguistique française $1: 141-159$.

Audétat M.C., Laurin S. \& Sanche G. (2011). « Aborder le raisonnement clinique du point de vue pédagogique. I. Un cadre conceptuel pour identifier les problèmes de raisonnement clinique », Pédagogie Médicale 12 : 223-229.

Auriac E. (2007). «Quels indicateurs retenir pour progresser dans l'étude des discours scolaires ? », in A. Sepcogna (dir.), Enseigner dans l'interaction. Paris : L'Harmattan, 33-56.

Auriac-Slusarczyk E. (2019). « Les discours entre soignants et patients. Études contemporaines. Introduction », ESASOS 5 (2) : 7-19.

Auriac-Slusarczyk E. \& Blasco M. (2019). Les discours soignants soignés adressés aux patients. Quelle contribution des sciences humaines et sociales? ESASOS 5(2).

Auriac-Slusarczyk E. \& Delsart A. (soumis). « Le lexique émotionnel et affectif en entretiens cliniques. Exploration du corpus DECLICS2016 », Tipa, avril 2020.

Auriac-Slusarczyk E. \& Delsart A. (à paraître). « Le lexique émotionnel et affectif en consultations médicales pour comprendre la relation de soin ", in M. Blasco, Parler à l'hôpital : écouter ce qui est dit, décrypter ce qui se dit. Munster : Nodus Editions.

Auriac-Slusarczyk E. \& Delsart A. (2020). « Le lexique émotionnel et affectif. Exploration du corpus DECLICS2016 », Colloque eMOTions, Bordeaux 4-5 juin 2020/report 26-27 novembre 2021.

Auriac-Slusarczyk E., Delsart A., Saint-Dizier V., Zehnder E., Blasco M., Advocat O., Durif F. \& Lebas-Fraczak L. (2018). «Étude pragmatique des discours soignants/soignés en contexte hospitalier ", $X^{e}$ Congrès francophone de psychologie de la santé, Pratiques et interventions en psychologie de la santé, 13-15 juin 2018. Metz : Université de Lorraine.

Balcou-Debussche M. \& Autier D. (2018). «Interventions éducatives et santé : éléments de problématisation », ESASOS 4(2) : 7-16.

Balcou-Debussche M. (2016). De l'éducation thérapeutique du patient à la littératie en santé. Problématisation socio-anthropologique d'objets didactiques contextualisés. Paris : Editions des Archives Contemporaines.

Balcou-Debussche M. (2016). «Interroger la littératie en santé en perspective de transformations individuelles et sociales. Analyse de l'évolution de 42 personnes diabétiques sur trois ans », Recherches \& Educations 16 : 73-87.

Batt M. \& Trognon A. (2012). Étude d'un jeu de dialogue professionnel. Le conseil en médecine prédictive. Nancy : éd. Maison des sciences de l'homme Lorraine, Collection Lang.

Berthod-Wurmser M., Bousquet F. \& Legal R. (2017). « Patients et usagers du système de santé : l'émergence progressive de voix qui commencent à compter ", Revue française des affaires sociales $1: 5-19$. 
Billon-Descarpentries J. (2000). « Essai de théorisation des modèles explicatifs de l'éducation appliquée à la santé », Spirale 25 : 17-30.

Blasco M. \& Cappeau P. (2018). « Construire et analyser un corpus oral sur objectifs spécifiques : précautions et réflexions ", Journées d'étude du Centre d'Études Linguistiques (CEL) et le Centre Interlangues Texte Image Langage, Corpus sur objectifs spécifiques, 15-16 novembre. Lyon : Université Jean Moulin.

Blasco M., Cappeau P., Auriac-Slusarczyk E., Advocat O., Delsart A., Richard E., Drouet G., Kébir Y. \& Saint Dizier De Almeida V. (2019). «DECLICS2016 : Un corpus pour recueillir, analyser et améliorer la parole en milieu hospitalier ", $10^{\text {es }}$ Journées Internationales de la Linguistique de corpus, 26-28 novembre. Grenoble : UGA.

Boelen C. (2014). « Il était une fois, il y a trente ans..., l'éducation médicale francophone », Pédagogie Médicale 15(1) : 61-62.

Bouacha A. (1981). « “Alors” dans le discours pédagogique: épiphénomène ou trace d'opérations discursives ", Langue Française 50 : 39-52.

Bousquet F. \& Ghadi V. (2017). « La participation des usagers aux systèmes de santé : un tour d'horizon international », Revue française des affaires sociales $1: 116-127$.

Cameron L. \& Deignan A. (2003). « Combining Large and Small Corpora to Investigate Tuning Devices Around Metaphor in Spoken Discourse », Metaphor and Symbol 18(3) : 149-160.

Chanet C. (2003), « Fréquence des marqueurs discursifs en français parlé : quelques problèmes de méthodologie », Recherches sur le français parlé $18:$ 1-25.

Charaudeau P. (1995). « Rôles sociaux et rôles langagiers », in D. Veronique \& R. Vion (dir.), Modèles de l'interaction verbale. Aix-en-Provence : Presses Universitaires de Provence, 79-96.

Chevalier G. (2007). « Les marqueurs discursifs réactifs dans une variété de française en contact intense avec l'anglais ", Langue française 154(2) : 61-77.

Chevalier G. (2008). « Les français du Canada : faits linguistiques, faits de langue », Alternative Francophone 1(1) : 80-97.

Clavurier V. (2014) «Les présentations cliniques. De la psychiatrie à la psychanalyse », Essaim 2 : 53-76.

Clot Y. (2007). « De l'analyse des pratiques au développement des métiers », Éducation \& Didactique 1(1) : 83-93.

Cosnier J., Grosjean M. \& Lacoste M. (1993). Les interactions en milieu soignant. Soins et communication: approches interactionnistes des relations de soin. Lyon : Presses universitaires de Lyon.

Côté L. (2015). « Réflexion sur une expérience de supervision clinique sous l'angle de l'alliance pédagogique ", Pédagogie Médicale 16(1) : 79-84.

Danino C. (2018). «Introduction. Les petits corpus », Corpus 18 : 1-10.

Delsart A. (2020). «Les statuts des locuteurs dévoilés par l'usage des répétitions locales en contexte de soin », Espaces Linguistiques $1: 26-4$.

Delsart A. \& Auriac-Slusarczyk E. (2020). « Étude pragmatique de la relation médecin/patient à partir de données orales authentiques ", $7^{e} e$ étition du Congrès Mondial de Linguistique Française, du 6 au 10 juillet. Montpellier 3 : Université Paul Valéry. 
Delsart A. \& Auriac-Slusarczyk E. (soumis). « Les emplois différentiels des marqueurs discursifs mais et donc en conversations cliniques : une étude linguistique appliquée à une pratique du soin éclairée ", Discours.

Delsart A. \& Marques A.-R. (2019). «Effet de l'expertise communicationnelle sur la prise de parole des patients. Étude contrastée des discours entre médecine et thérapeutes ", ESASOS 5(2) : 59-83.

Do M. \& Bissieres C. (2018). « L'observance à l'épreuve du soin éducatif : la posture de patient réflexif en question », Les dossiers des Sciences de l'Education 39 : 71-88.

Emanuel E.-J. \& Emanuel L.-L. (1992). « Four models of the physician-patient relationship », JAMA 267(16) : 2221-2226.

Ericsson K.A. (2004). « Deliberate Practice and the Acquisition and Maintenance of Expert Performance in Medicine and Related Domains ", AcadMed 79(10 suppl.) : 70-81.

Fainzang S. (2006). « Secret et mensonge dans le discours médical », Cités 2(6) : 27-36.

Ferré G. (2016a). « Between Fact and Fiction: Semantic fields and Image Content in Crime Infotainment programs », Multimodal Communication 5(2) : 127-141.

Ferré G. (2016b). « Gesture/speech integration in the perception of prosodic emphasis ", $9^{\text {th }}$ International Conference on Speech Prosody, 13-16 June 2018. Poland : Poznan.

Fournier C. \& Kerzanet S. (2007). « Communication médecin-malade et éducation du patient, des notions à rapprocher : apports croisés de la littérature ", Santé Publique 19(5) : 413-425.

Freidson E. (1984). La profession médicale. Paris : Éditions Payot.

Gaillard M. \& Lechopier N. (2015). « Relever le défi d'introduire aux sciences humaines et sociales en première année commune des études de santé. Mise en perspective de quelques pratiques pédagogiques ", Pédagogie Médicale 16 : 23-34.

Génolini J.-P., Roca R., Rolland C. \& Mambrado M. (2011). « "L'éducation” du patient en médecine générale : une activité périphérique ou spécifique de la relation de soin?", Revue sciences sociales et santé $3: 81-12$.

Henrion-Latché J. \& Auriac-Slusarczyk E. (2020). « Étude sémantico-pragmatique du discours au profit d'un décryptage du parcours psychologique suivi d'adolescents fragilisés. Proposition d'une démarche de codage de discours ", Corpus $20: 1-20$.

Jouquan J., Parent F. \& Audétat M.-C. (2013). « Des analogies entre le raisonnement médical et l'évaluation formative ", Revue française de linguistique appliquée XVIII(1) : 93-106.

Kébir Y., Delsart A., Arfaoui S., Auriac-Slusarczyk E. \& Saint-Dizier de Almeida V. (2020). «L'apport d'une approche pluridisciplinaire pour enrichir l'analyse de l'activité de consultation de suivi médicale ». Colloque de la SELF, L'activité et ses frontières. Penser et agir sur les transformations de nos sociétés. 16-18 septembre. Paris : La villette.

Kerbrat-Orecchioni C. (2001). « 'Je voudrais un p'tit bifteck', La politesse à la française en site commercial », Les Cahiers du Cédisor 7 : 105-118.

Kerbrat-Orecchioni C. (2004). « Négocier dans les petits commerces », Négociations 2(2) : 7-22.

Kerbrat-Orrecchioni C. (1987). « La mise en places », in J. Cosnier \& C. Kerbrat-Orecchioni (dir.). Décrire la conversation. Lyon : Presses Universitaires de Lyon, 319-352.

Louis-Courvoisier M. (2015). « Pertinence des sciences humaines et sociales en médecine », Pédagogie Médicale 16(1) : 5-8. 
Lussier M.-T. \& Richard C. (2008). « Because one shoes doesn't fit all : A repertoire of doctorpatient relationships ", Canadian family psysician/Medecin de famille canadien 54(8) : 1089-1092.

Marchand P. (1998). L'analyse du discours assisté par ordinateur. Paris : A. Colin.

Mayaffre, D. (2012). Nicolas Sarkozy. Mesure et démesure du discours (2007-2012). Paris : Presses de la fondation nationale des Sciences Politiques.

Mayaffre, D. (2017). « Les mots des candidats, de "allons" à "vertu” ", in P. Perrineau (dir.). Le vote disruptif. Les élections présidentielles et législatives de 2017. Paris : Presses de Sciences Po, Collection Chroniques électorales, 129-152.

Mayaffre D., Pincemin B. \& Poudat C. (2019). « Explorer, mesurer, contextualiser. Quelques apports de la textométrie à l'analyse de discours », Langue française 203(3) : 101-115.

Mellet S. (2002). « Corpus et recherches linguistiques, Introduction », Corpus 1:1-6.

Millette B., Lussier M.-T. \& Goudreau J. (2004). «L'apprentissage de la communication par les médecins : aspects conceptuels et méthodologiques d'une mission académique prioritaire ", Pédagogie Médicale 5(2) : 110-126.

Moumjid N. \& Carrère M.-O. (2000). «La relation médecin-patient, l'information et la participation des patients à la décision médicale : les enseignements de la littérature internationale ", Revue française des affaires sociales 2(2) : 73-88.

Nguyen-Khac A. (2017). « Mesures de l'accès aux soins : l'apport d'enquêtes sur les pratiques et perceptions des patients ", Revue française des affaires sociales $1: 187-195$.

Piot T. (2018). « Le rôle déterminant et méconnu des conversations soignant-patient au cœur des interactions de soin ", Les dossiers des sciences de l'éducation 39 : 13-31.

Préau M. \& Siméone A. (2018). De l'expertise scientifique à l'expertise profane. Lyon : Psychologie du social. Éditions des archives contemporaines.

Richard C., Lussier M.-T., Galarneau S. \& Jamoulle O. (2010). « Compétence en communication professionnelle en santé ", Pédagogie Médicale 11(4) : 255-272.

Roiné P., Blasco M. \& Auriac-Slusarczyk E. (à paraître). « Rôle et valeurs des emplois en "c'est" dans le corpus Philosophèmes ", in $\mathrm{C}$. Frérot et $\mathrm{M}$. Pecman, Des corpus numériques à la modélisation linguistique en langues de spécialité. Grenoble : Presses de l'UGA, pages non connues.

Saint-Dizier de Almeida V. (2013). « Comment améliorer la compréhension de l'entretien d'annonce de diagnostics médicaux sérieux », Activités 10(2) : 54-81.

Sommer J.-M. \& Rieder A.-R. (2014). « Comment enseigner la communication au cabinet », PrimaryCare 14(8) : 136-138.

Szasz T. S. \& Hollender M. H. (1956). « The Basic Models of the Doctor-Patient Relationship », Archives of Internal Medicine 97 : 585-592.

Thievenaz J. (2018). « Les situations d'apprentissage réciproques (le cas de la consultation médicale) », Les dossiers des sciences de l'éducation 39 : 131-150.

Trognon A. \& Larrue J. (1997). Pragmatique du discours politique. Paris : A. Colin.

Trognon A. (1995). « Pragmatique formelle et pragmatique empirique », L'information grammaticale $66: 17-24$.

Trognon A. (2012). « Connexions dans l'histoire de la logique interlocutoire », Connexions 2: 83-98.

Turpin B. (2002). « Le jargon figure du multiple », La linguistique 38(1) : 53-68. 
Vincent D. (1993). Les ponctuants de la langue et autres mots du discours. Québec : Éditions Nuit Blanche.

\section{ANNEXES}

\section{ANNEXE 1}

\begin{tabular}{|c|c|c|c|c|}
\hline Auteurs & \multicolumn{4}{|c|}{ Modèles de relation médecin-patient } \\
\hline Parsons et al., 1951 & Actif / Passif & & & \\
\hline Szasz \& Hollender, 1956 & Actif / Passif & $\begin{array}{l}\text { Actif / Actif* } \\
* \quad \text { participation } \\
\text { mutuelle }\end{array}$ & Passif / Passif & Passif / Actif \\
\hline $\begin{array}{l}\text { Freidson, } 1984 \text { (sociologie } \\
\text { interactionniste) }\end{array}$ & $\begin{array}{l}\text { Médecin } \\
\text { coopératif }\end{array}$ & Patient guide & Patient actif & $\begin{array}{l}\text { Médecin } \\
\text { passif }\end{array}$ \\
\hline Emanuel \& Emanuel, 1992 & Informatif & Interprétatif & $\begin{array}{l}\text { Délibératif* } \\
* \quad \text { Idéal } \\
\text { auteurs }\end{array}$ & Paternaliste \\
\hline Moumjid \& Carrère, 2000 & $\begin{array}{l}\text { Patient } \\
\text { décideur }\end{array}$ & & $\begin{array}{l}\text { Décision } \\
\text { partagée }\end{array}$ & $\begin{array}{l}\text { Médecin } \\
\text { décideur }\end{array}$ \\
\hline
\end{tabular}

Tableau 3. Modèles de relation médecin-patient dans la littérature reproduit d'après Fournier \& Kerzanet, 2007 et Moumjid \& Carrère, 2000.

\section{ANNEXE 2}


Image 1022DEB400002E760000304F24557713ADFA23DE.emf

Figure 1. The possible transformations in the doctor-patient relationship: Type of relationship is determined by probiem ond health care context.

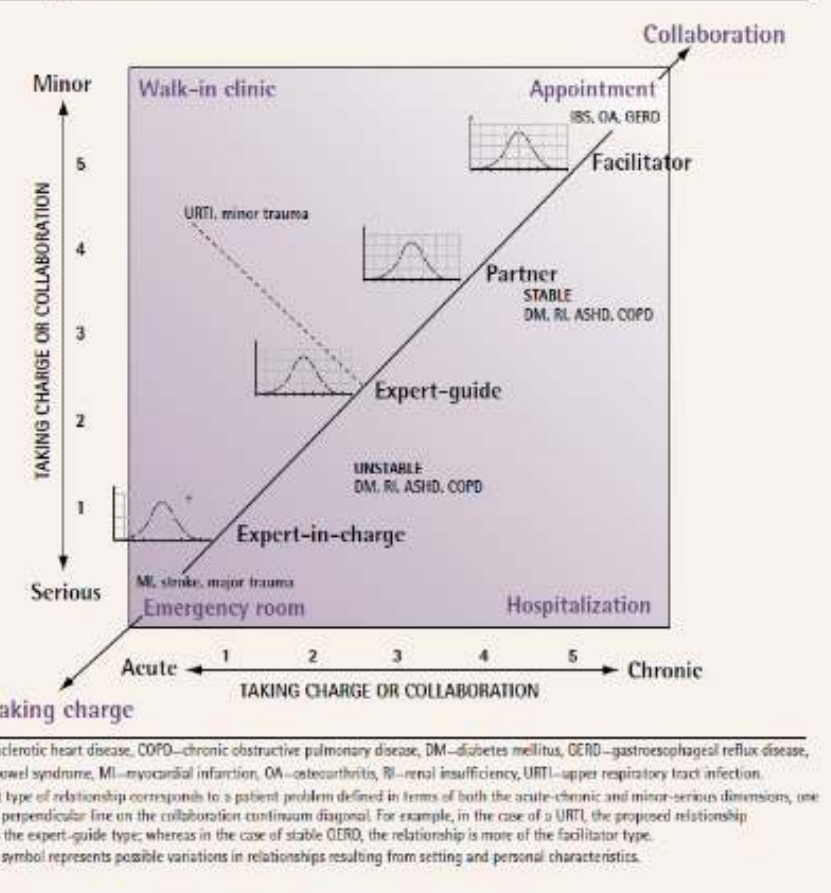

Figure extraite de Lussier \& Richard, 2008, p. 1090.

ANNEXE 3. Corpus DECLICS2016 au 3 avril 2020

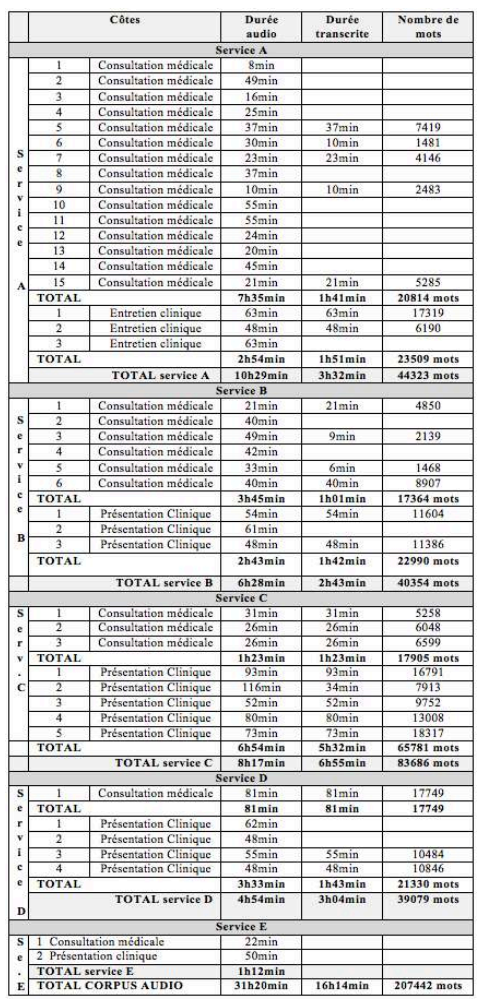


Légende. Une consultation médicale se déroule entre un médecin et un patient. Une présentation clinique se déroule entre un thérapeute et un patient déjà vu par son médecin en présence d'un auditoire silencieux (cf. Charcot). Un entretien clinique se déroule entre un thérapeute et un patient déjà vu par son médecin.

\section{ANNEXE 4}

Vignette 1 : Monsieur X (PAT) accompagné de son épouse Madame Z aidante (AID) s'entretiennent avec Madame Y médecin spécialiste (MED) pour la consultation régulière de suivi survenant 2 mois après une opération de Monsieur X. Des symptômes gênants et persistants apparaissent pouvant donner lieu à échange dans cette consultation.

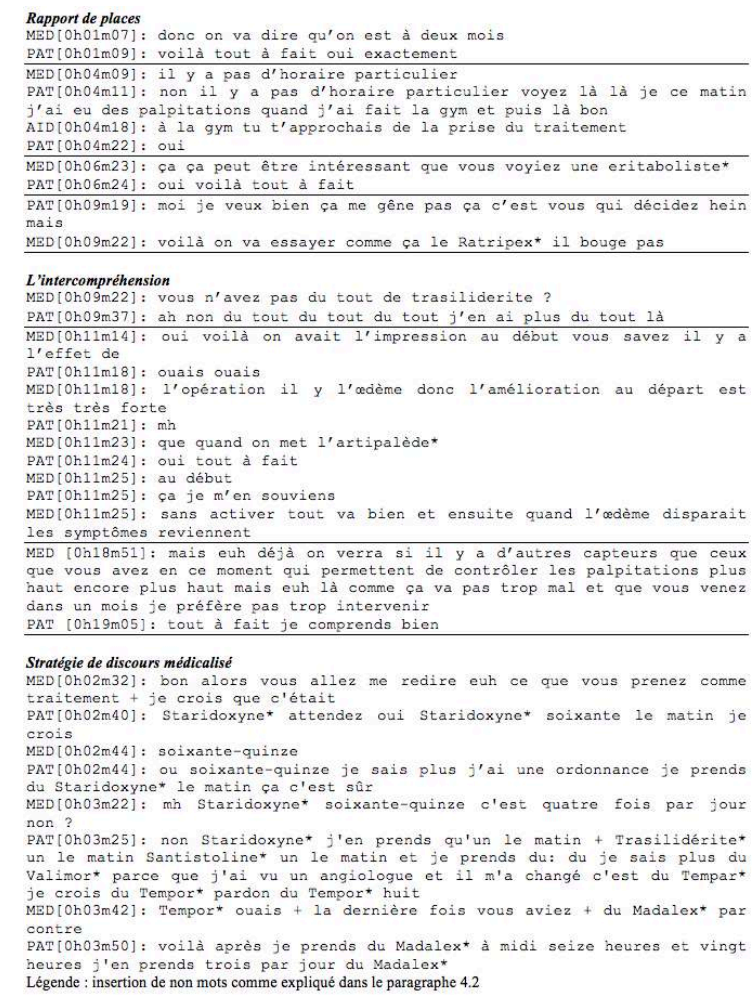

Vignette 2 : Monsieur V (PAT) consulte le médecin spécialiste W (MED) pour une consultation de suivi afin d'abaisser le nombre de traitements et de réduire le nombre de spécialistes qui suivent Monsieur V. 


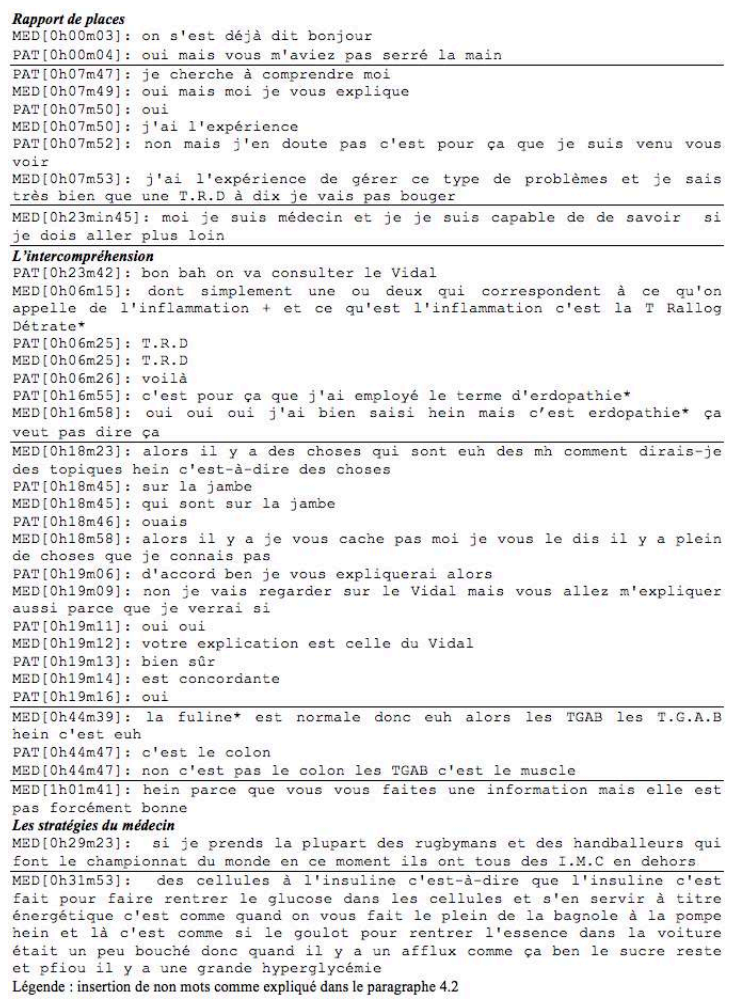

Vignette 3 : Madame U (PAT) est suivie par la médecin spécialiste T (MED) pour des problèmes hormonaux.

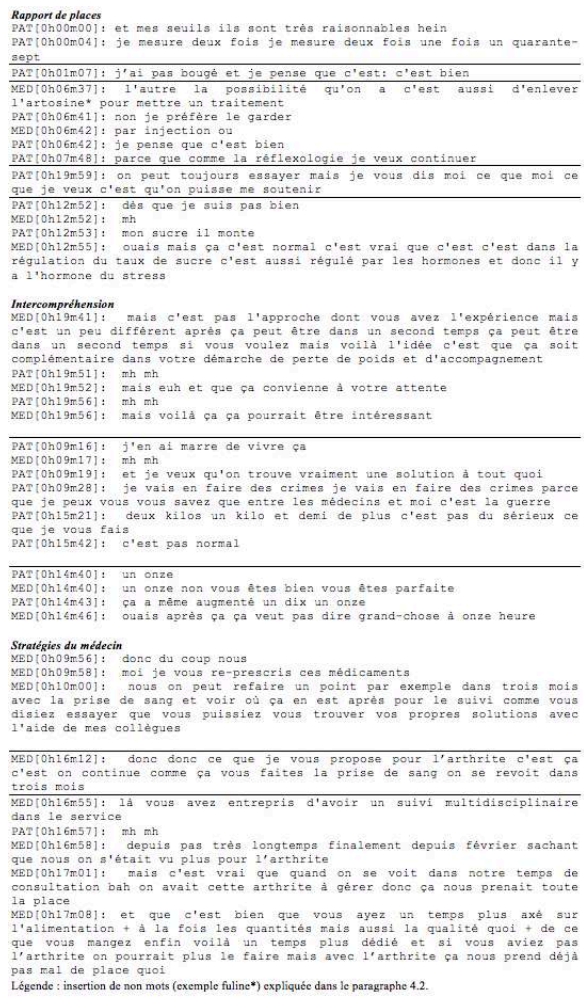




\section{NOTES}

1. Le projet DECLICS, qui regroupe des chercheurs en national, est financé par la région AURA.

2. Nom générique désignant le corpus verbal.

3. L'enregistrement, exclusivement en mode audio, protège néanmoins déjà le caractère personnel des données; mais l'anonymisation reste incomplète.

4. Voir la plateforme Multiplumes de la MSH Clermont-Auvergne dédiée: http://mshclermont.fr/content/multiplumes-incubateur-de-projets-corpus-pour-les-shs.

5. Les chercheurs intéressés peuvent adresser une demande à mylène.blasco@uca.fr, qui pourra leur être accordée sous couvert d'un respect de confidentialité pour partager ces données scientifiques, ce, avec accès restrictif. Toute demande sera préalablement soumise et examinée par le comité éthique de l'UCA.

6. Chevalier utilise 6 corpus, respectivement de $20000,84600,177900,530000,210000$ et 35000 mots.

7. Chanet utilise les corpus CORPAIX, 1050000 mots et CRFP 450000 mots.

8. La transcription croise notamment les conventions du GARS (français parlé) et de VALIBEL (interaction).

9. Les extraits de corpus présentés aux médecins ont été nettoyés de toutes marques de convention de transcription qui pourraient gêner la lecture, seules les pauses courtes, symbolisées avec le signe classique «+ », sont maintenues.

10. Informations correspondant à l'état du 03 avril 2020.

11. Nous soulignons minimalement les pronoms personnels en partie indicateurs des rapports de places interlocutives.

12. Propos tronqués ou glosés.

13. Une lecture silencieuse précédait une lecture orale intonative proche de l'enregistrement effectif pour coller à l'authenticité du contexte.

14. Idem. Propos partiellement tronqués pour préserver le choix de non divulgation des médecins (voir § 4.5.).

15. Accord du CERES de Paris-Descartes acquis sur demande du 10/12/2016 par Mylène Blasco et Océane Advocat.

\section{RÉSUMÉS}

Notre proposition engage à (re)problématiser l'intérêt de la linguistique de et sur corpus quant à ses applications professionnelles. Ancrée en pragmatique, elle retrace le mode de recueil et d'exploitation de données dans le cadre applicatif du corpus DECLICS2016, ce, pour servir les questions de métier en médecine. Nous testons l'efficience de la linguistique, comme discipline de SHS contributive éclairant l'activité professionnelle, en produisant des scénarios pédagogiques à partir d'extraits de discours. On s'attache à réfléchir sur la « reconstruction de la relation médecin patient ", à partir d'un matériau de paroles authentiques. En premier lieu, l'accent est porté sur la constitution même du corpus DECLICS2016. En second lieu, c'est l'application en santé qui est interrogée via nos résultats. Ceux-ci dégagent, à partir d'entretiens de confrontation croisée entre médecins mis face aux paroles authentiques et données d'études linguistiques de patients, qu'il est délicat, pour ces médecins, de profiter de nos catégories 
pragmatiques prédéterminées: rapports de place, intercompréhension et stratégie discursive du médecin.

The aim of our proposal is to ask questions and better consider the opportunity of speech data applying to professional field. Inscribed in pragmatic way, our presentation describes work into progress to data collected linked to applicative framework of DECLICS2016 corpus, to engage professional medical questions. We test the linguistic efficiency, considered as one of SHS discipline, to highlight medical activity with pedagogical scenarios, on the base of discursive examples. We encourage think about the possibility of "reconstructing relationships" between specialist doctors and their patients, based on authentic original speech acts. Firstly, we take into account the strategy of constitution DECLICS corpus. Secondly, our results are looked to benefit healthcare area. This results show that, based on self- crossed interview between doctors, in front of original sentences and linguistics patient's data, these specialist doctors do not progress with our pragmatic categorization assistance: relationships, understanding and medical discourse strategies.

\section{INDEX}

Keywords : relationships, pedagogical scenarios, constitution of corpus, pragmatic

Mots-clés : rapports de place, scénarios pédagogiques, constitution de corpus, pragmatique

\section{AUTEURS}

\section{EMMANUĖLE AURIAC-SLUSARCZYK}

Maître de conférences HDR en Sciences du Langage

Université Clermont Auvergne, ACTé, F-63 000 Clermont -Ferrand, France

\section{ALINE DELSART}

Doctorante en Sciences du Langage

Université Clermont Auvergne, ACTé, F-63 000 Clermont -Ferrand, France 